\title{
Association of Prenatal anxiety with adverse pregnancy outcomes - a prospective hospital based study.
}

\author{
Dr Arun Kumar Dwivedi, Asst Prof (Psychiatry), Army College of Medical Sciences, New Delhi, India
}

Background : Anxiety is common in pregnant women and known increase towards the third trimester. ${ }^{[1]}$ Studies in the past have shown an association of prenatal anxiety with adverse pregnancy outcomes such as low birth weight, lower APGAR scores, emergency caesarean deliveries and instrument deliveries, although the results are conflicting. [2],[3],[4] Hence this study was undertaken to evaluate effect of anxiety on pregnancy outcomes.

Materials and Methods: The study was cleared by the institutional ethics committee. Two hundred randomly selected consenting registered pregnant women reporting for antenatal follow up during third trimester of pregnancy were included in the study. Hindi version of Perinatal Anxiety Screening scale (PASS) was used to assess anxiety. These women were followed up in the post-natal period to assess pregnancy outcomes (which included Caesarian sections including emergency Caesarian sections, Instrumental deliveries, Post-partum haemorrhage $(\mathrm{PPH})$, birth asphyxia and low birth weight). Students t-test, Chi-square test, ANOVA and correlation coefficients were calculated.

Results : Analysis was conducted on 195 subjects. Most women (48.7\%) were between 26 and 30 yrs of age. Primigravidas comprised $11.3 \%$ of total, while $22.6 \%$ reported a past abortion. Bad obstetric history was reported by $10.4 \%$ of the women, while $20.5 \%$ reported suffering from hypothyroidism. Mean anxiety score was 23.6 (range 5-80). Adverse pregnancy outcomes (as defined in the study) was noted in 99 women (50.76\%), however anxiety scores in this group were not different from the group without adverse outcomes. Surprisingly, the group with lowest scores was that of emergency caesarean section. Further, no correlation was found between PASS scores and other parameters.

Discussion : Perinatal anxiety scores were lower (subthreshold) and did not affect the pregnancy outcomes. The result is contrary to existing literature.
Surprisingly, the group with lowest scores was that of emergency caesarean section. The reasons for such result could be improved medical care, greater satisfaction and trust and better overall mental health of the women undergoing follow up at this centre.

Conclusion : Prenatal anxiety, as assessed using Perinatal anxiety screening scale was not found to be associated with adverse pregnancy outcomes. This finding is contrary to the results of earlier studies. More enquiry is needed into this area to replicate the results with clarity in larger samples.

Conflict of interest : There are no conflicts to disclose. No funds were obtained for the conduct of the study. The study was not funded by any agency and was supported by the hospital in which the study was carried out.

\section{References}

1. Loomans EM, Van Dijk AE, Vrijkotte TG, Van Eijsden M, Stronks K, Gemke RJ, et al. Psychosocial stress during pregnancy is related to adverse birth outcomes: results from a large multi-ethnic community-based birth cohort. Eur J Public Health. 2012;23(3):485-491.

2. Yali AM, Lobel M. Coping and distress in pregnancy: an investigation of medically high risk women. $\mathrm{J}$ Psychosom Obstet Gynecol. 1999;20(1):39-52.

3. Nasreen HE, Kabir ZN, Forsell Y, Edhborg M. Low birth weight in offspring of women with depressive and anxiety symptoms during pregnancy: results from a population based study in Bangladesh. BMC Public Health. 2010;10(1):515.

4. Zhu P, Tao F, Hao J, Sun Y, Jiang X. Prenatal life events stress: implications for preterm birth and infant birthweight. Am J Obstet Gynecol. 2010;203(1):34-e1.

PASS (Perinatal Anxiety Screening Scale) SCORE

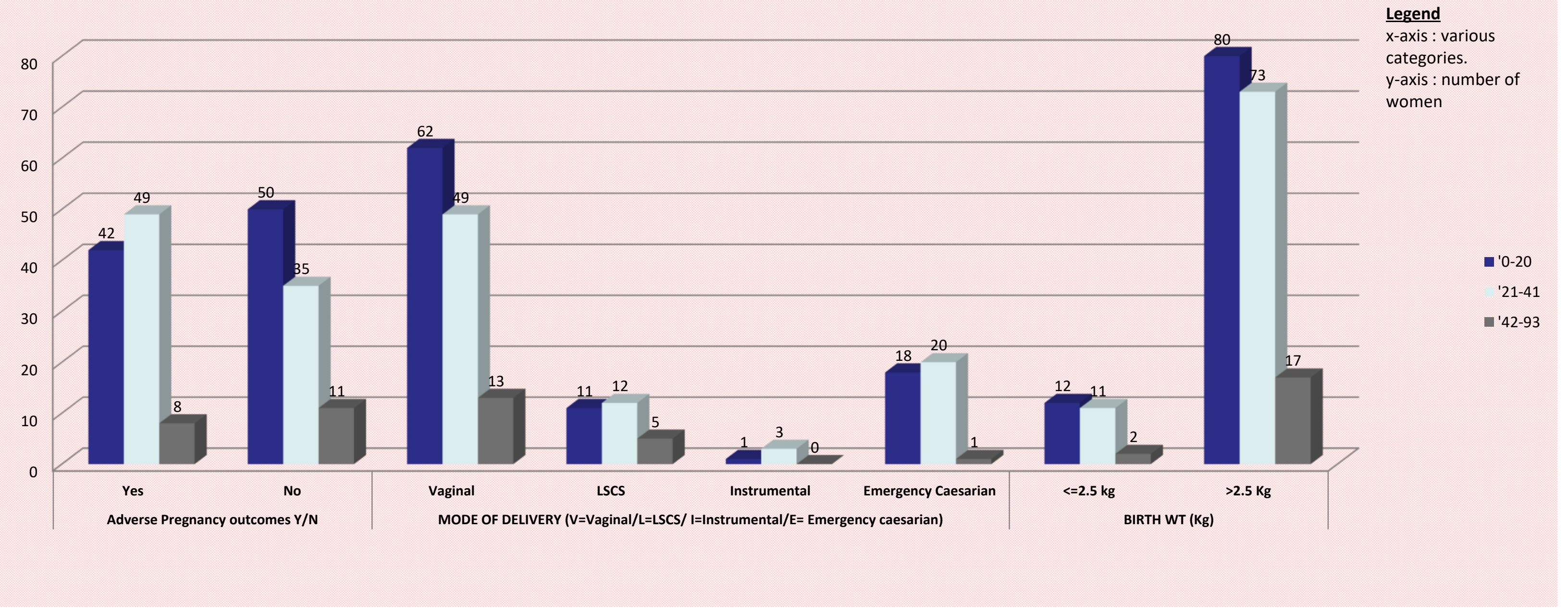

Figure 1. - showing Number of women in each group showing mild, moderate and severe anxiety as per PASS scores. 